\title{
Toward a Theory of Planarity: Hanani-Tutte and Planarity Variants
}

\author{
Marcus Schaefer \\ School of Computing, DePaul University, Chicago, IL 60604, USA \\ mschaefer@cs . depaul . edu
}

\begin{abstract}
We study Hanani-Tutte style theorems for various notions of planarity, including partially embedded planarity, and simultaneous planarity. This approach brings together the combinatorial, computational and algebraic aspects of planarity notions and may serve as a uniform foundation for planarity, as suggested in the writings of Tutte and Wu.
\end{abstract}

\section{Introduction}

Planarity of graphs is a well-understood topic, but as soon as we modify it in any one of many different ways such as allowing crossings, clustering vertices, requiring monotone drawings, simultaneously drawing multiple graphs, or extending a partially embedded graph, we very quickly lose the ground under our feet; some problems become NP-complete (upward planarity, book embeddability), for others feasible algorithmic solutions are unknown (c-planarity, simultaneous planarity).

In 1972 Tutte published his paper "Toward a Theory of Crossing Numbers" in which he suggested an algebraic treatment of crossing numbers 1 The classic Hanani-Tutte theorem states that a graph is planar if and only if it can be drawn in the plane so that no two independent edges cross oddly, where two edges are independent if they do not share an endpoint. We rephrase this as a crossing number result: given a drawing $D$ of $G$, let $\operatorname{iocr}(D)$ be the number of pairs of independent edges of $G$ that cross oddly in $D$. Then $\operatorname{iocr}(G)$ is the minimum of iocr $(D)$ over all drawings of $G$. We say a drawing $D$ of $G$ is iocr-0 if $\operatorname{iocr}(D)=0$. We call an edge in a drawing (independently) even if it crosses every other (independent) edge an even number of times.

Theorem 1 (Hanani-Tutte). A graph $G$ is planar if and only if $\operatorname{iocr}(G)=0$.

This theorem implies an algebraic characterization of planarity, since the condition $\operatorname{iocr}(G)=0$ can be rephrased as a system of linear equations over $\mathrm{GF}(2)$ leading to a simple polynomial time algorithm for planarity testing, as is well-known.

In this paper we begin a systematic study of whether and how the HananiTutte theorem extends to variants of planarity. The theorem turns out to be very versatile and adaptable, giving rise to a uniform approach to many of the variants of planarity considered in the literature. Table 1 summarizes known and new results on Hanani-Tutte theorems for planarity notions.

\footnotetext{
1 There were precursors to his approach, notably the paper by Hanani [12, but also work by Flores, van Kampen, and Wu. Some of the history can be found in 33 .
} 
Table 1. Summary of known and new results on planarity variants

\begin{tabular}{|c|c|c|c|c|}
\hline \multirow[b]{2}{*}{ planarity notion } & \multirow[b]{2}{*}{ recognition } & \multirow[b]{2}{*}{ obstr. } & \multicolumn{2}{|c|}{ Hanani-Tutte } \\
\hline & & & result & algorithm \\
\hline standard & linear [24] & 27 & $\begin{array}{llll}12 & 35 \\
\end{array}$ & folklore \\
\hline outer & linear [24] & 10 & & \\
\hline partially embedded & linear [3] & 25 & Thm 3 & Rem 2 \\
\hline partial rotation & linear, Cor 1 & open & Thm 4 & in $\mathbf{P}$ \\
\hline partial rotation (with flips) & in $\mathbf{P}$, Cor 3 & open & open & open \\
\hline$x$-monotone & linear [26] & open & \begin{tabular}{|lll|l|}
28 & 29 & 18 \\
\end{tabular} & quadratic [18] \\
\hline level & linear [26] & open & $\begin{array}{l}\text { implicit in } \\
18\end{array}$ & quadratic [18 \\
\hline radial level & linear $[6]$ & open & open & open \\
\hline upward & NPC [19] & & & \\
\hline projective & linear & 2115 & 31 & open \\
\hline book & $\begin{array}{l}\mathbf{N P C}[13] \\
\text { special cases } \\
\text { in } \mathbf{P}\end{array}$ & & special cases & special cases \\
\hline$c$ (clustered) & open & open & open & open \\
\hline$c l$ (clustered level) & open & open & open & open \\
\hline simultaneous & $\begin{array}{l}\text { special cases } \\
\text { in } \mathbf{P}\end{array}$ & open & $\begin{array}{l}\text { special cases } \\
\text { Thm } 5\end{array}$ & $\begin{array}{l}\text { special cases in } \\
\mathbf{P}\end{array}$ \\
\hline
\end{tabular}

Section 2 contains a detailed sketch of how to derive a Hanani-Tutte theorem for partially embedded planarity, and Theorem 5 in Section 3 announces several Hanani-Tutte theorems for special cases of simultaneous planarity; the general case remains open. Because of space limitations, full details of most results and the underlying redrawing tools have to be left for the journal version of this paper. However, we include a conjecture that gives a flavor of the type of redrawing involved.

Conjecture 1. Suppose a graph $G$ with subgraph $H$ can be drawn so that every edge of $H$ crosses every independent edge evenly. Then there is a drawing of $G$ in which edges of $H$ do not cross each other, and there are no new pairs of independent edges crossing oddly.

The truth of this conjecture would imply a single polynomial-time recognition algorithm for nearly all known planarity variants, including the infamous $c$ planarity. The reason is two-fold: first, it implies a polynomial time algorithm for simultaneous planarity of two graphs (by Corollary 2). Secondly, many planarity variants reduce to the simultaneous planarity of two graphs, so all such variants could then be decided in polynomial time 2 We include two examples of

\footnotetext{
${ }^{2}$ Problem $A$ reduces to problem $B$ if there is a polynomial-time computable function $f$ so that $x \in A$ if and only if $f(x) \in B$. This notion of reduction is very weak: for example, $c$-planarity reduces to book embeddability, since $c$-planarity lies in NP and book embeddability is NP-complete. However, we cannot (directly) read off a $c$-planar embedding of $G$ from a book embedding of $f(G)$. Our reductions will be "natural" in that an embedding of $f(G)$ will encode an embedding of $G$.
} 
connections between different planarity variants: Theorem 6 reduces $c$-planarity to simultaneous planarity, and Lemma 3 reduces embeddability of a graph with partial rotation system with flips allowed to a case of simultaneous planarity that can be solved in polynomial time. Figure 1 summarizes known and new reductions between planarity notions.

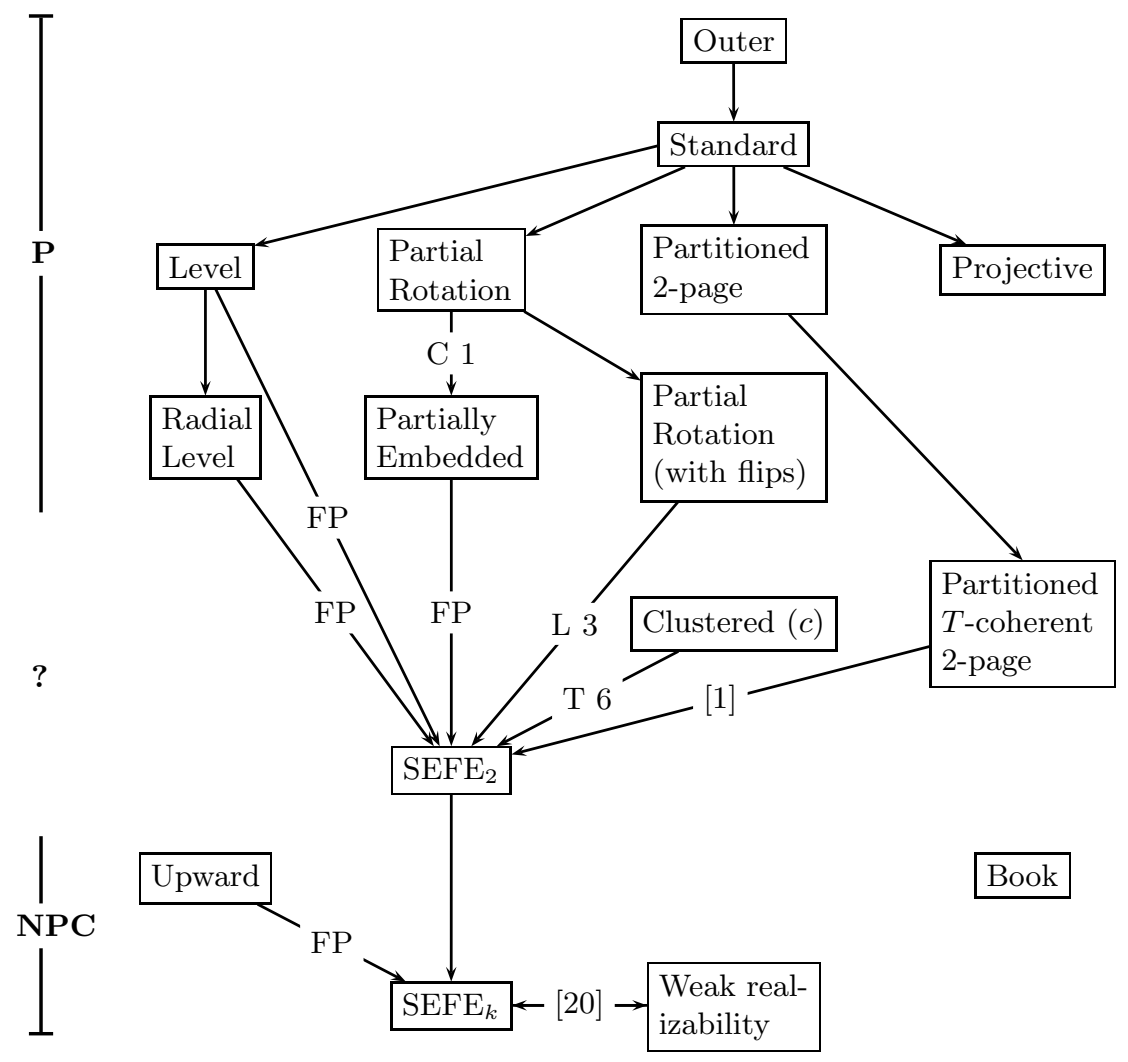

Fig. 1. A directed edge denotes a natural reduction between two problems. Reductions for which no reference is given are folklore or straightforward; FP means that the result will be in the full paper.

Algorithmically, the algebraic approach cannot compete with PQ-trees and SQPR-trees which give linear-time algorithms in many cases. It does, however, lead to algorithmic solutions in some cases where no other algorithms are currently available, and it deepens our theoretical understanding of planarity.

We only define the main planarity variants used in this paper; for additional definitions see, for example, Maurizio Patrignani's recent survey in the "Handbook of Graph Drawing" 34]. 


\section{Partially Embedded Planarity}

A partially embedded graph $(P E G)(G, H, \mathcal{H})$ consists of a graph $G$, a subgraph $H$ of $G$, and an embedding $\mathcal{H}$ of $H$ in the plane. We consider two embeddings topologically equivalent if there is an orientation-preserving homeomorphism of the plane that takes one to the other. A PEG $(G, H, \mathcal{H})$ is planar if there is a planar embedding of $G$ that contains $\mathcal{H}$. If $\mathcal{H}$ and $\mathcal{H}^{\prime}$ are two topologically equivalent embeddings of $H$, then $(G, H, \mathcal{H})$ is planar if and only if $\left(G, H, \mathcal{H}^{\prime}\right)$ is planar, so we can redraw $\mathcal{H}$ as long as we maintain topological equivalence. Partially embedded planarity can be tested in linear time using SPQR-trees, a result due to Angelini, Di Battista, Frati, Jelínek, Kratochvíl, Patrignani, and Rutter [3]. If $\mathcal{H}$ is a straight-line embedding, and we require $G$ to be a straightline embedding of $\mathcal{H}$, the problem is NP-complete (Patrignani [30]).

Remark 1. If $H$ is not connected, it can have many embeddings that are not topologically equivalent, but that all have the same rotation system. See Theorem 4 for a weakening of partially embedded planarity that captures only the rotation system.

Jelínek, Kratochvíl, and Rutter 25] give a forbidden obstruction characterization of partially embedded graphs. Since we are dealing with partially embedded graphs $(G, H, \mathcal{H})$, the usual minor operations need some modification; for example, we cannot arbitrarily contract edges in $E(G)-E(H)$ since the effect on $\mathcal{H}$ can be ambiguous, and there are new operations available to us: if we delete an edge in $H$, do we delete it in $G$ as well? Without going into the details here, it is sufficient to note that the paper by Jelínek, Kratochvíl, and Rutter [25] contains a definition of a $P E G$ minor for which they can identify a well-behaved (if not finite) obstruction set. We retain the names $D_{i}$ and $A_{k}$ of the graphs from that paper, without defining the actual graphs.

Theorem 2 (Jelínek, Kratochvíl, Rutter [25]). A PEG-graph $(G, H, \mathcal{H})$ is planar if and only if it does not contain any of the obstructions $K_{5}, K_{3,3}$, or $D_{1}$, $D_{2}, D_{3}, D_{4}, D_{11}, D_{14}, D_{16}, D_{17}$ or $A_{k}, k \geq 3$ as a PEG-minor.

As far as Hanani-Tutte is concerned, the relevant facts about PEG-minors and the obstruction set can be summarized as follows. Recall that a drawing of a graph is iocr-0 if every two independent edges cross each other an even number of times.

Lemma 1. (i) Suppose $(G, H, \mathcal{H})$ is a PEG-minor of $\left(G^{\prime}, H^{\prime}, \mathcal{H}^{\prime}\right)$ and there is an iocr-0 drawing of $G^{\prime}$ extending $\mathcal{H}^{\prime}$. Then there is an iocr-0 drawing of $G$ extending $\mathcal{H}$. (ii) None of the obstructions identified in Theorem 2 has an iocr-0 drawing.

Combining those two results gives us the Hanani-Tutte theorem for partially embedded planarity. 
Theorem 3. Suppose we are given a graph $G$ and a planar embedding $\mathcal{H}$ of a subgraph $H \subseteq G$. Then $G$ has a planar embedding that extends $\mathcal{H}$ if and only if there is an iocr-0 drawing of $G$ containing $\mathcal{H}$.

We hope to give a direct proof of Theorem 3 at some point, avoiding obstruction sets.

Remark 2. The Hanani-Tutte theorem for partially embedded planarity given in Theorem 3 can be turned into a polynomial-time algorithm. This algorithm is no competition to the linear-time algorithm by Angelini, Di Battista, Frati, Jelínek, Kratochvíl, Patrignani, and Rutter [3].

When we ask whether a PEG $(G, H, \mathcal{H})$ is planar we start with a fixed planar embedding $\mathcal{H}$ of $H$. What if we only have a rotation system for $H$, or even weaker than that, a partial rotation system? Say $\rho$ is a partial rotation system for a graph $G$ if $\rho$ specifies a cyclic order of a subset $E_{v}^{\rho}$ of the edges $E_{v}$ incident to $v$ for every vertex $v$. Specifying a partial rotation system is more general than specifying the rotation system of a subgraph $H$, since the partial rotation system need not fix both ends of an edge in their respective rotations.

Let $\operatorname{cr}(G, \rho)$ be the minimum $\operatorname{cr}(D)$ over all drawings $D$ of $G$ that respect the partial rotation system $\rho$, where we say that $D$ respects $\rho$ if the cyclic rotation of edges $E_{v}^{\rho}$ at $v$ is as prescribed by $\rho$. For $\operatorname{iocr}(G, \rho)$ we use a modified definition which also counts odd crossings between adjacent edges if they are part of the same $E_{v}^{\rho}$. Given a drawing $D$ respecting $\rho$, we define $\operatorname{iocr}(D, \rho):=\operatorname{iocr}(D)+$ $\sum_{v \in V(H)} \sum_{e, f \in E_{v}^{\rho}}\left(i_{D}(e, f) \bmod 2\right)$. Let iocr $(G, \rho)$ be the minimum of $\operatorname{iocr}(D, \rho)$ where $D$ ranges over all drawings of $G$ respecting $\rho$.

Theorem 4. If $\operatorname{iocr}(G, \rho)=0$ then $\operatorname{cr}(G, \rho)=0$.

From this theorem we can obtain an algebraic criterion for testing whether a graph $G$ has an embedding respecting a partial rotation system $\rho$, but it is easier to just use the reduction from embedding $(G, \rho)$ to partially embedded planarity which is implicit in the proof of Theorem 4 .

Corollary 1. Embeddability of a graph with partial rotation system reduces to partially embedded planarity.

Since partially embedded planarity can be rested in linear time using the algorithm of Angelini, Di Battista, Frati, Jelnek, Kratochvl, Patrignani, and Rutter 4], embeddability of graphs with partial rotation systems can be tested in linear time as well.

\section{Simultaneous Graph Drawing}

A simultaneous drawing of two graphs $G_{i}=\left(V\left(G_{i}\right), E\left(G_{i}\right)\right), i \in\{1,2\}$, is a drawing of $G_{1} \cup G_{2}=\left(V\left(G_{1}\right) \cup V\left(G_{2}\right), E\left(G_{1}\right) \cup E\left(G_{2}\right)\right.$. It is a simultaneous 
embedding with fixed edges (SEFE) of the two graphs if the drawings of $G_{1}$ and $G_{2}$ considered by themselves are planar embeddings. In other words, if any two edges $e$ and $f$ cross in the drawing, we must have $e \in E\left(G_{1}\right)-E\left(G_{2}\right)$ and $f \in E\left(G_{2}\right)-E\left(G_{1}\right)$ or vice versa 3 We also say $\left(G_{1}, G_{2}\right)$ is, or $G_{1}$ and $G_{2}$ are, simultaneously planar.

Simultaneous planarity generalizes to arbitrarily many graphs, and we will use $\mathrm{SEFE}_{k}$ if we want to emphasize that we are referring to that version. Testing whether three or more graphs are simultaneously planar is known to be NPcomplete [20]. The complexity of simultaneous planarity for two graphs remains open, but it is tempting to conjecture that it is in $\mathbf{P}$. In fact, we will state a (combinatorial) conjecture later which implies that simultaneous planarity is in P. Several special cases have been solved recently. One can test whether $\left(G_{1}, G_{2}\right)$ is simultaneously planar

(i) in linear time if $G_{1} \cap G_{2}$ is 2-connected (Haeupler, Jampani, Lubiw [22 23]),

(ii) in linear time if $G_{1} \cap G_{2}$ consists of disjoint cycles (Bläsius, Rutter [9]),

(iii) in quadratic time if a fixed embedding of each connected component of $G_{1} \cap G_{2}$ is given (Bläsius, Rutter [9]),

(iv) in quadratic time if $G_{1}$ and $G_{2}$ are 2-connected, and $G_{1} \cap G_{2}$ is connected (Bläsius, Rutter [8]).

Results on more than two graphs are rarer, though Angelini, Di Battista, and Frati showed that we can test in polynomial time whether 3 embedded graphs have a simultaneous embedding that respects the original embeddings; for more than 14 graphs, the problem is NP-complete [2]. For a survey on simultaneous planarity, see [7].

We can establish Hanani-Tutte style theorems for simultaneous planarity in the following cases:

(a) $G_{1} \cap G_{2}$ consists of disjoint 2-connected components and isolated vertices,

(b) one of $G_{1}$ or $G_{2}$ is the disjoint union of subdivisions of 3-connected graphs,

(c) $G_{1} \cap G_{2}$ is subcubic (maximal degree at most 3).

Theorem 5. If $\operatorname{siocr}\left(G_{1}, G_{2}\right)=0$ and $(a),(b)$ or $(c)$ holds, then $\operatorname{scr}\left(G_{1}, G_{2}\right)=0$.

It follows that there are polynomial-time algorithms for testing simultaneous planarity in all three cases. These generalize the results listed under $(i)$ and $(i i)$ above.

We also give algebraic characterizations of simultaneous planarity if $G_{1} \cap G_{2}$ has a fixed embedding or each connected component of $G_{1} \cap G_{2}$ has a fixed embedding, giving an alternative polynomial time algorithm for (iii) above. At this point, we do not know whether $(i v)$ has an algebraic characterization, though we suspect it does.

3 The qualification "with fixed edges" refers to the fact that every edge belonging to both graphs, a common edge, is represented by the a single curve. For a simultaneous embedding without fixed edges there is no requirement to draw common edges as the same curve. It is generally agreed that the nomenclature is unfortunate, but it has become common. 
In Section 3.2 we will see how to apply case $(b)$ of Theorem 5 to test embeddability of graphs with a partial rotation system where flipping of rotations is allowed.

\subsection{Simultaneous Planarity and Hanani-Tutte}

For a simultaneous drawing $D$ of $\left(G_{1}, G_{2}\right)$, we let $D\left[G_{i}\right]$ be the drawing $D$ restricted to the edges in $G_{i}$. The simultaneous crossing number, $\operatorname{scr}\left(G_{1}, G_{2}\right)$, as the minimum of $\operatorname{cr}\left(D\left[G_{1}\right]\right)+\operatorname{cr}\left(D\left[G_{2}\right]\right)$ over all simultaneous drawings $D$ of $G_{1}$ and $G_{2}$ as introduced by Chimani, Jünger and Schulz 11 . So $\operatorname{scr}\left(G_{1}, G_{2}\right)=0$ if and only if $\left(G_{1}, G_{2}\right)$ is simultaneously planar. Similarly, we define $\operatorname{siocr}\left(G_{1}, G_{2}\right)$, the simultaneous independent odd crossing number as the minimum of $\operatorname{iocr}\left(D\left[G_{1}\right]\right)+$ $\operatorname{iocr}\left(D\left[G_{2}\right]\right)$ over all simultaneous drawings $D$ of $G_{1}$ and $G_{2}$. We say a simultaneous drawing $D$ of $G_{1}$ and $G_{2}$ is $\operatorname{siocr}-0$ if $\operatorname{iocr}\left(D\left[G_{1}\right]\right)+\operatorname{iocr}\left(D\left[G_{2}\right]\right)=0$.

Our main conjecture is a Hanani-Tutte result for simultaneous embeddability of two graphs.

Conjecture 2. If $\operatorname{siocr}\left(G_{1}, G_{2}\right)=0$, then $\operatorname{scr}\left(G_{1}, G_{2}\right)=0$.

If the conjecture were true, then testing for simultaneous planarity would be in polynomial time since the condition $\operatorname{siocr}\left(G_{1}, G_{2}\right)=0$ can be written as a system of linear equations over $\mathrm{GF}(2)$.

Corollary 2. If Conjecture 2 is true, then simultaneous planarity of two graphs can be decided in polynomial time.

Conjecture 2 is specifically about simultaneous drawings, but one can bring the problem back to drawings of single graphs, namely Conjecture 1 which we saw earlier.

Lemma 2. If Conjecture 1 is true, then so is Conjecture 2.

Proof. Suppose $\operatorname{siocr}\left(G_{1}, G_{2}\right)=0$ and let $H=G_{1} \cap G_{2}$ and $G=G_{1} \cup G_{2}$; we can assume that $V(H)=V(G)$ by adding any vertices in $V(G)-V(H)$ to $V(H)$. $G$ and $H$ fulfill the assumptions of Conjecture 1, so if that conjecture is true, we can find a drawing of $G$ in which edges of $H$ no longer cross each other, and there are no new independent pairs of edges crossing oddly. In other words, an siocr-0 drawing of $G_{1}$ and $G_{2}$ containing an embedding $\mathcal{H}$ of $H$ (if considered by itself). The siocr-0 drawing of $\left(G_{1}, G_{2}\right)$ can be restricted to an iocr-0 drawing of PEG $\left(G_{1}, H, \mathcal{H}\right)$ and an iocr-0 drawing of PEG $\left(G_{2}, H, \mathcal{H}\right)$. By Theorem 3 there are planar drawings of $G_{1}$ and $G_{2}$ that extend $\mathcal{H}$. Since $\mathcal{H}$ contains all the vertices of $G_{1}$ and $G_{2}$, the two drawings together are a simultaneous planar drawing of $G_{1}$ and $G_{2}$, thus establishing the (conditional) truth of Conjecture 2

The truth of Conjecture 2 would establish SEFE as a benevolent incarnation of the weak realizability problem, and an important one, since, just like the weak realizability it is powerful enough to encode many other graph drawing problems. The conjecture is open, but Theorem 5 shows that several special cases can be settled. 


\subsection{Simultaneous Planarity and Rotation Systems}

Corollary 1 showed that embeddability of a graph $G$ with a partial rotation system $\rho$ reduces to partial embedded planarity. In this section we want to add one small twist: we allow rotations at certain vertices to flip, that is, we specify a set $U \subseteq V(G)$ so that for each vertex $v \in U$ the cyclic order of edges $E_{u}^{\rho}$ at $u$ is either as specified by $\rho$ or reversed. Partially embedded planarity is not flexible enough to capture this variant, but simultaneous planarity is.

Lemma 3. Given a graph $G$ with partial rotation system $\rho$ and a set of vertices $U \subseteq V(G)$, the problem of whether $G$ can be embedded so that the rotation at all vertices is as specified by $\rho$, with flips allowed for vertices in $U$, reduces to a simultaneous planarity problem, where one of the graphs is the disjoint union of 3-connected components.

Proof. Let $G_{1}$ be $G$ after each edge has been subdivided twice; in particular, $V(G) \subseteq V\left(G^{\prime}\right)$. With each edge $v u$ in $E_{v}^{\rho}$ we can uniquely associate an edge in $G^{\prime}$ : pick the first edge on the $P_{3}$ from $v$ to $u$ in $G^{\prime}$. Turn the star of edges with center $v$ whose rotation is determined by $\rho$ into a wheel, so that the additional cycle respects the rotation at $v$. Add all these wheels to a new graph $G_{2}$. A simultaneous embedding of $\left(G_{1}, G_{2}\right)$ contains a subdivision of $G$ which realizes $\rho$ except that the rotation at every vertex may be flipped. Take all the wheels in $G_{2}$ associated with vertices belonging to $V(G)-U$, and add edges to that subgraph until it is triangulated; subdivide each of the new edges once (so we do not create common edges with $G_{1}$ ); add all the new edges and vertices to $G_{2}$. Now in a simultaneous embedding of $\left(G_{1}, G_{2}\right)$ either all rotations of vertices in $V(G)-U$ are flipped compared to $\rho$ or none of them are. Vertices associated with vertices in $U$, however, can still flip independently.

Corollary 3. Suppose we are given a graph $G$ with partial rotation system $\rho$ and a set of vertices $U \subseteq V(G)$. We can test in polynomial time whether $G$ can be embedded so that the rotation at all vertices is as specified by $\rho$, except for vertices of $U$ where rotations are also allowed to fip.

Proof. The result follows immediately from Lemma 3 and the fact that Theorem 5 implies a polynomial-time algorithm for case $(b)$.

\section{Clustered Planarity}

Planarity of clustered graphs entered the graph drawing literature in papers by Feng, Cohen and Eades [17/16] under the name $c$-planarity 4 We base the following definition on the one given by Cortese and Di Battista in their very readable survey paper [14.

A clustered graph is a graph $G=(V, E)$ together with a rooted tree $T$ whose leaves are the vertices of $G$. Every internal vertex $\nu$ of $T$ corresponds to the

${ }^{4}$ Feng's thesis $[15$. makes it clear that $c$-planarity was meant to abbreviate compound planarity, but it has generally been taken to mean clustered planarity. 
cluster $V(\nu)$, the set of vertices of $G$ occurring as leaves below $\nu$ in $T$. Let $G(\nu)$ be the subgraph of $G$ induced on $V(\nu)$ (not necessarily connected). A drawing of a clustered graph $(G, T)$ is a drawing of $G$ so that for every internal vertex $\nu$ of $T$ there is a (connected) region $R(\nu)$ of the plane so that: $G(\nu)$ is drawn inside $R(\nu)$ and $R(\nu) \subseteq R(\mu)$ if and only if $\nu$ is a descendant of $\mu$ in $T$. We say an edge $e$ is incident to $\nu$ if $e$ has one endpoint in $V(\nu)$ and the other in $V-V(\nu)$. An edge-region crossing occurs if an edge $e$ that is not incident to $\nu$ crosses the boundary of $R(\nu)$ or if an edge $e$ that is incident to $\nu$ crosses the boundary of $R(\nu)$ more than once. A drawing of a clustered graph is c-planar if it contains no edge crossings and no edge-region crossings.

\section{Theorem 6. c-planarity reduces to the SEFE problem.}

Proof. Suppose we are given a clustered graph $(G, T)$; without loss of generality, we can assume that for every $v \in V(G)$ there is a vertex $\nu$ of $T$ so that $V(\nu)=\{v\}$ (each vertex is contained in its own region). We have to create two graphs $G_{1}$ and $G_{2}$ so that $(G, T)$ is $c$-planar if and only if $G_{1}$ and $G_{2}$ have a simultaneous embedding with fixed edges. We will use $G_{1}$ to ensure a planar drawing of $G$ and both $G_{1}$ and $G_{2}$ to enforce the clustering constraints. We start by letting $G_{1}=G$ (this will change during the construction).

For every cluster $V(\nu)$, where $\nu$ is an interior vertex of $T$, we split all edges of $G_{1}$ incident to $\nu$ into two halves. We then add the region gadget $C_{\nu}$ as shown in Figure 2 and connect the severed ends to corresponding vertices in the gadget (if one end is connected to $u_{i}$, the other end is connected to $v_{i}$ ). Note that $C_{\nu}$ contributes edges to both $G_{1}$ and $G_{2}$. If $\mu$ is a descendant of $\nu$ in $T$ we add a $G_{2}$ edge from the inner hook in $C_{\nu}$ to the outer hook in $C_{\mu}$. If $\mu$ is a leaf of $T$, so $V(\nu)$ consists of a single vertex, we add a $G_{1}$ edge from $u_{1}$ to $w_{I}$.

If $(G, T)$ is $c$-planar, then $G_{1}$ and $G_{2}$ have a simultaneous embedding with fixed edges, indeed, we can construct such an embedding in parallel to how we constructed $G_{1}$ and $G_{2}$ from $(G, T)$. For the reverse direction, we let $R_{\nu}$ be the gray area of $C_{\nu}$; let $S_{\nu}$ be the triangular (gray) subarea of $R_{\nu}$. First note that every vertex of $V(\nu)$ lies in $S_{\nu} \subset R_{\nu}$ : since every vertex was enclosed in its own region, there is a path from each vertex $u \in V(\nu)$ to $w_{I}$ consisting of $G_{2}$ and $G_{1} \cap G_{2}$ edges only (since we hooked up the region gadgets via $G_{2}$ edges). But a path of $G_{2}$ edges cannot cross the boundaries of $S_{\nu}$, so $v$ has to lie inside that triangle. From $G_{1}$ we can recover an embedding of $G$ : we move $u$ and $v$ together into the center of the black $G_{1}$-edge between them and split the vertices into parallel edges, reconnecting the original ends. This is possible, since the gate gadget as part of the region gadget enforces that the ordering of the $u_{i}$ is the same as of the $v_{i}$.

\section{Beyond Planarity}

While planarity and its variants are important parts of graph drawing, in practice many visualization tasks will require allowing crossings of some type. This is already built into some of the drawing models: simultaneous planarity pointedly 


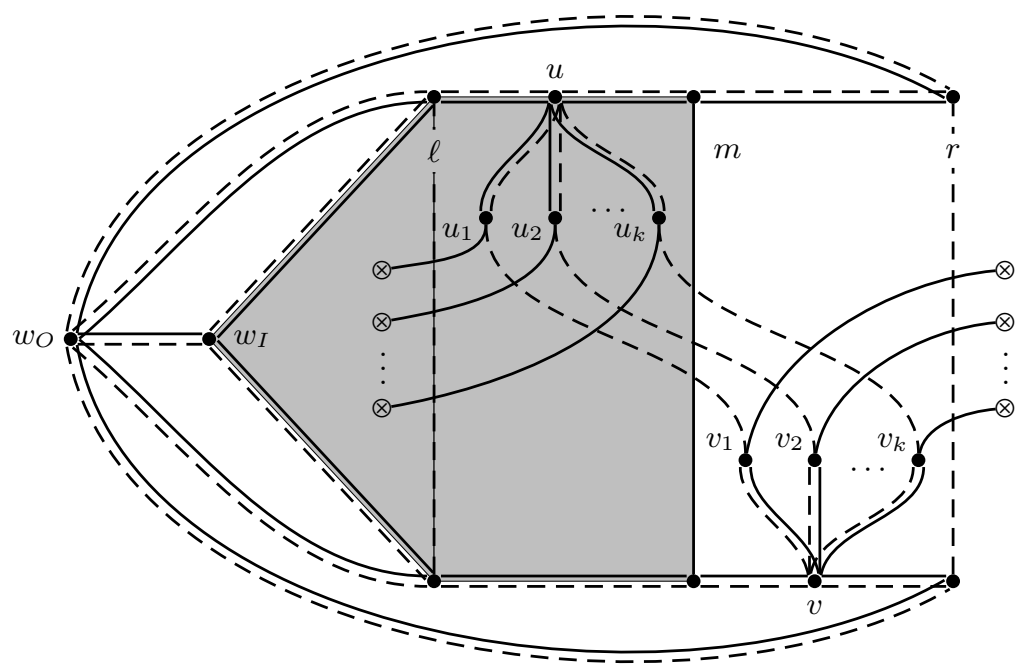

Fig. 2. The region gadget $C_{\nu}$ drawn in the case that $\nu$ is incident to $k$ edges. The connector half-edges terminate in a $\otimes$. The inner hook is $w_{I}$, the outer hook $w_{O}$. The drawing of any gadgets corresponding to a $\mu$ which is a descendant of $\nu$ are forced to lie in the triangle in the gray region. We will choose $R_{\nu}$ to be the gray area of $C_{\nu}$.

ignores certain types of crossings in the drawing, and weak realizability gives full flexibility (at the expense of NP-completeness).

In a next step, we should consider crossing number variants. We already saw that the Hanani-Tutte theorem can be stated as $\operatorname{iocr}(G)=0$ implies that $\operatorname{cr}(G)=$ 0. Indeed, $\operatorname{iocr}(G)=\operatorname{cr}(G)$ as long as $\operatorname{iocr}(G) \leq 2$ and $\operatorname{cr}(G) \leq\left(\begin{array}{c}2 \operatorname{iocr}(G) \\ 2\end{array}\right)$ [32, and there are graphs for which $\operatorname{iocr}(G)<\operatorname{cr}(G)$.

Question 1. Is there a function $f$ for which $\operatorname{scr}\left(G_{1}, G_{2}\right) \leq f\left(\operatorname{siocr}\left(G_{1}, G_{2}\right)\right)$ ?

Acknowledgment. Thanks to Michael Pelsmajer for his thorough comments.

\section{References}

1. Angelini, P., Di Battista, G., Frati, F., Patrignani, M., Rutter, I.: Testing the Simultaneous Embeddability of Two Graphs Whose Intersection Is a Biconnected Graph or a Tree. In: Iliopoulos, C.S., Smyth, W.F. (eds.) IWOCA 2010. LNCS, vol. 6460, pp. 212-225. Springer, Heidelberg (2011)

2. Angelini, P., Di Battista, G., Frati, F.: Simultaneous Embedding of Embedded Planar Graphs. In: Asano, T., Nakano, S.-I., Okamoto, Y., Watanabe, O. (eds.) ISAAC 2011. LNCS, vol. 7074, pp. 271-280. Springer, Heidelberg (2011)

3. Angelini, P., Di Battista, G., Frati, F., Jelínek, V., Kratochvíl, J., Patrignani, M., Rutter, I.: Testing planarity of partially embedded graphs. In: Charikar, M. (ed.) SODA 2010, pp. 202-221. SIAM (2010) 
4. Angelini, P., Di Battista, G., Frati, F., Jelínek, V., Kratochvíl, J., Patrignani, M., Rutter, I.: Testing planarity of partially embedded graphs. In: Charikar, M. (ed.) Proceedings of the Twenty-First Annual ACM-SIAM Symposium on Discrete Algorithms, SODA 2010, Austin, Texas, USA, January 17-19, pp. 202-221. SIAM (2010)

5. Archdeacon, D.: A Kuratowski theorem for the projective plane. J. Graph Theory 5(3), 243-246 (1981)

6. Bachmaier, C., Brandenburg, F.J., Forster, M.: Radial level planarity testing and embedding in linear time. J. Graph Algorithms Appl. 9(1), 53-97 (electronic) (2005)

7. Bläsius, T., Kobourov, S.G., Rutter, I.: Simultaneous Embeddings of Planar Graphs. In: Tamassia, R. (ed.) Handbook of Graph Drawing and Visualization. CRC Press (to appear)

8. Bläsius, T., Rutter, I.: Simultaneous PQ-ordering with applications to constrained embedding problems. CoRR abs/1112.0245 (2011)

9. Bläsius, T., Rutter, I.: Disconnectivity and Relative Positions in Simultaneous Embeddings. ArXiv e-prints (April 2012)

10. Chartrand, G., Harary, F.: Planar permutation graphs. Ann. Inst. H. Poincaré Sect. B (N.S.) 3, 433-438 (1967)

11. Chimani, M., Jünger, M., Schulz, M.: Crossing minimization meets simultaneous drawing. In: Visualization Symposium, PacificVIS 2008, pp. 33-40. IEEE (2008)

12. Chojnacki (Haim Hanani), C.: Über wesentlich unplättbare Kurven im dreidimensionalen Raume. Fundamenta Mathematicae 23, 135-142 (1934)

13. Chung, F.R.K., Leighton, F.T., Rosenberg, A.L.: Embedding graphs in books: a layout problem with applications to VLSI design. In: Graph Theory with Applications to Algorithms and Computer Science (Kalamazoo, Mich., 1984), pp. 175-188. Wiley-Intersci. Publ., Wiley, New York (1985)

14. Cortese, P.F., Di Battista, G.: Clustered planarity. In: Computational Geometry, SCG 2005, pp. 32-34. ACM, New York (2005)

15. Feng, Q.W.: Algorithms for Drawing Clustered Graphs. Ph.D. thesis, Department of Computer Science and Software engineering, University of Newclastle (April 1997)

16. Feng, Q.W., Cohen, R.F., Eades, P.: How to Draw a Planar Clustered Graph. In: Li, M., Du, D.-Z. (eds.) COCOON 1995. LNCS, vol. 959, pp. 21-30. Springer, Heidelberg (1995)

17. Feng, Q.W., Cohen, R.F., Eades, P.: Planarity for Clustered Graphs. In: Spirakis, P.G. (ed.) ESA 1995. LNCS, vol. 979, pp. 213-226. Springer, Heidelberg (1995)

18. Fulek, R., Pelsmajer, M., Schaefer, M., Štefankovič, D.: Hanani-Tutte, monotone drawings, and level-planarity. In: Pach, J. (ed.) Thirty Essays on Geometric Graph Theory, pp. 263-287. Springer (2012)

19. Garg, A., Tamassia, R.: On the computational complexity of upward and rectilinear planarity testing. SIAM J. Comput. 31(2), 601-625 (electronic) (2001)

20. Gassner, E., Jünger, M., Percan, M., Schaefer, M., Schulz, M.: Simultaneous Graph Embeddings with Fixed Edges. In: Fomin, F.V. (ed.) WG 2006. LNCS, vol. 4271, pp. 325-335. Springer, Heidelberg (2006)

21. Glover, H.H., Huneke, J.P., Wang, C.S.: 103 graphs that are irreducible for the projective plane. J. Combin. Theory Ser. B 27(3), 332-370 (1979)

22. Haeupler, B., Jampani, K.R., Lubiw, A.: Testing Simultaneous Planarity When the Common Graph Is 2-Connected. In: Cheong, O., Chwa, K.-Y., Park, K. (eds.) ISAAC 2010, Part II. LNCS, vol. 6507, pp. 410-421. Springer, Heidelberg (2010) 
23. Haeupler, B., Jampani, K., Lubiw, A.: Testing simultaneous planarity when the common graph is 2-connected. CoRR abs/1009.4517 (2010)

24. Hopcroft, J., Tarjan, R.: Efficient planarity testing. J. Assoc. Comput. Mach. 21, 549-568 (1974)

25. Jelínek, V., Kratochvíl, J., Rutter, I.: A Kuratowski-type theorem for planarity of partially embedded graphs. In: Hurtado, F., van Kreveld, M.J. (eds.) SoCG 2011, pp. 107-116. ACM (2011)

26. Jünger, M., Leipert, S., Mutzel, P.: Level Planarity Testing in Linear Time. In: Whitesides, S.H. (ed.) GD 1998. LNCS, vol. 1547, pp. 224-237. Springer, Heidelberg (1998)

27. Kuratowski, C.: Sur les problèmes des courbes gauches en Topologie. Fund. Math. 15, 271-283 (1930)

28. Pach, J., Tóth, G.: Monotone drawings of planar graphs. J. Graph Theory 46(1), 39-47 (2004)

29. Pach, J., Tóth, G.: Monotone drawings of planar graphs. ArXiv e-prints (January 2011)

30. Patrignani, M.: On extending a partial straight-line drawing. Internat. J. Found. Comput. Sci. 17(5), 1061-1069 (2006)

31. Pelsmajer, M.J., Schaefer, M., Stasi, D.: Strong Hanani-Tutte on the projective plane. SIAM Journal on Discrete Mathematics 23(3), 1317-1323 (2009)

32. Pelsmajer, M.J., Schaefer, M., Štefankovič, D.: Removing independently even crossings. SIAM Journal on Discrete Mathematics 24(2), 379-393 (2010)

33. Schaefer, M.: Hanani-Tutte and related results, to appear in Bolyai Memorial Volume

34. Tamassia, R.: Handbook of Graph Drawing and Visualization. Discrete Mathematics and Its Applications. Chapman and Hall (2012) (to appear)

35. Tutte, W.T.: Toward a theory of crossing numbers. J. Combinatorial Theory 8 , 45-53 (1970) 